\title{
New chaps in the histone chaperone arena
}

\author{
Eric I. Campos and Danny Reinberg ${ }^{1}$ \\ Department of Biochemistry, Howard Hughes Medical Institute, New York University School of Medicine, \\ New York 10016, USA
}

\begin{abstract}
Understanding exactly how chromatin is assembled is paramount to addressing how select histone modifications may be transmitted, a putative epigenetic process. In the June 15, 2010, issue of Genes \& Development, Drané and colleagues (pp. 1253-1265) identified DAXX as a novel $\mathrm{H} 3.3$-specific chaperone. This finding, in the context of others published by Goldberg and colleauges in Cell and Sawatsubashi and colleagues (pp. 159-170) in the January 15, 2010, issue of Genes \& Development, provides the impetus for uncovering the mechanistic and functional properties of alternative histone deposition pathways.
\end{abstract}

\section{H3 histone variants and associated chaperones}

Canonical nucleosomes are comprised of 147 base pairs (bp) of DNA folded around a histone octamer. The central tetramer, comprising two H3-H4 dimers, is flanked by two separate H2A-H2B dimers (Luger et al. 1997). In mammals, the $(\mathrm{H} 3-\mathrm{H} 4)_{2}$ tetramer includes either of three noncentromeric somatic H3 variants-namely, H3.1, $\mathrm{H} 3.2$, and H3.3-and the invariant histone H4. The first two H3 variants differ by a single amino acid and are considered to be replication-coupled (RC), since they are expressed during S phase (Hake and Allis 2006). The latter differs by four additional amino acids from $\mathrm{H} 3.1$, is expressed throughout the cell cycle (Hake and Allis 2006), and is considered replication-independent (RI). Despite being nearly identical (Fig. 1), these histone variants differ in genomic localization (Goldberg et al. 2010) and global post-translational modifications (PTMs) (Loyola et al. 2006), and correlate with different levels of transcription (Jin et al. 2009; Goldberg et al. 2010).

Affinity purification of exogenous H3.1 and H3.3 histones revealed distinct histone variant-specific chaperone complexes (Tagami et al. 2004). The RC H3.1 histone copurified with CAF-1 (Tagami et al. 2004), a trimeric histone chaperone. CAF-1 is tethered to the proliferating cell nuclear antigen (PCNA) (Shibahara and Stillman 1999) and couples H3.1 deposition to newly synthesized DNA behind the replication fork, as well as to repair

[Keywords: Histone variant; H3.3; histone chaperone; PML-NBs] ${ }^{1}$ Corresponding author.

E-MAIL reinbd01@nyumc.org; FAX (212) 263-9040.

Article is online at http://www.genesdev.org/cgi/doi/10.1101/gad.1946810. synthesis following DNA damage (Shibahara and Stillman 1999; Moggs et al. 2000). On the other hand, the RI H3.3 was found to associate with the HIRA chaperone, which is capable of assembling chromatin independently of replication in somatic cells (Tagami et al. 2004), as well as in the male pronucleus of fertilized Drosophila eggs prior to the first round of replication (Loppin et al. 2005).

Despite the exclusivity of the $\mathrm{H} 3.1$ variant to mammals, evidence suggests that both RC and RI assembly processes are conserved throughout evolution and proceed in a stepwise manner. ASF1, an $\mathrm{H} 3-\mathrm{H} 4$ chaperone, is hypothesized to buffer and transmit soluble newly synthesized histones to both CAF-1 and HIRA during chromatin assembly through direct chaperone-chaperone interactions (Tyler et al. 2001; Mello et al. 2002; Tagami et al. 2004; Green et al. 2005). Of note, ASF1 strictly binds $\mathrm{H} 3-\mathrm{H} 4$ dimers by masking the $\mathrm{H} 3-\mathrm{H} 3$ ' interface that is responsible for the formation of heterotypic $(\mathrm{H} 3-\mathrm{H} 4)_{2}$ tetramers (English et al. 2006; Natsume et al. 2007). Thus, CAF-1 and HIRA function by acting downstream from the chaperone ASF1, and are thought to assemble (H3$\mathrm{H} 4)_{2}$ tetramers during deposition (Fig. 2).

In a surprising twist, two previously unsuspected proteins, DAXX and DEK, have now been found capable of chaperoning H3.3 histones independently of HIRA. The groups of Ali Hamiche (Drané et al. 2010) and David Allis (Goldberg et al. 2010) confirmed that DAXX-a protein associated previously with FAS-mediated apoptosis-is physically associated with ATRX, a member of the SWI2/ SNF2-like ATP-dependent chromatin remodelers (Xue et al. 2003). However, the death-associated protein 6 (DAXX), which is specific to the animal kingdom and shares homology with the yeast Rtt106 histone chaperone in an acidic domain, is now shown to promote the deposition of H3.3-H4 units (Drané et al. 2010). The targeting of the H3.3 variant by DAXX seems to be partially dependent on ATRX, which directs H3.3 deposition at genomic locations distinct from those of HIRA (Drané et al. 2010; Goldberg et al. 2010; Wong et al. 2010).

Through an innovative genome editing technology, involving engineered zinc finger modules coupled to an endonuclease to induce homologous recombination at a specific locus (Carroll 2008), Allis and colleagues (Goldberg et al. 2010) were able to specifically tag one allele of the endogenous H3.3 gene, thereby averting artificial overexpression. By further mutating this gene to express the H3.2 variant, the approach resulted in a thorough and 


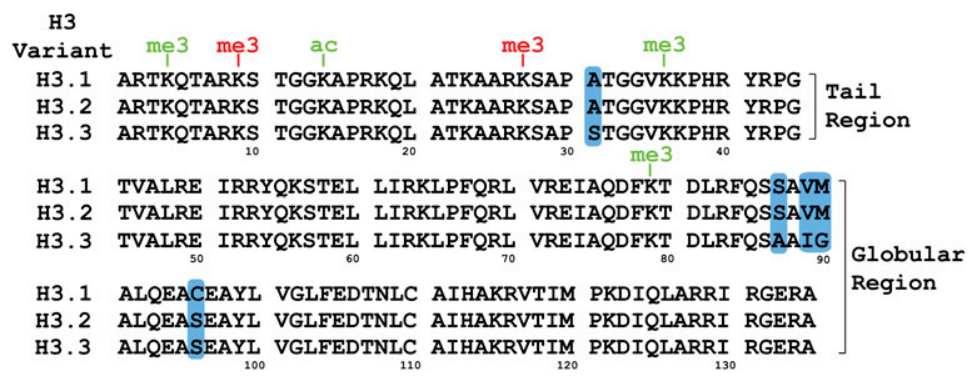

Figure 1. Sequence alignment of human canonical H3 variants. No substitutions are found within key residues known to facilitate transcriptional silencing or activation. Changes in amino acid composition are highlighted in blue. Modifications generally correlating with active transcription are shown in green, and those correlating with silenced chromatin are shown in red. discriminating survey of genome-wide H3.2 and H3.3 distributions in wild-type or chaperone-deficient ESCs (embryonic stem cells). H3.3 was enriched at the direct vicinity of the transcription start sites of genes with high CpG content promoters, regardless of gene activity, but also within the body of active genes (Goldberg et al. 2010; Jin et al. 2010). However, this enrichment was HIRAdependent, as H3.3 was now absent in protein-coding regions in HIRA-deficient cells. Unexpectedly, H3.3 was still deposited at most transcription factor-binding sites (TFBSs), and, intriguingly, transcription levels were sustained in HIRA-null ESCs (Goldberg et al. 2010).
This finding redirects how H3.3 is viewed in the context of transcription. Exactly where must $\mathrm{H} 3.3$ reside to engender transcription competency? Is it sufficient that H3.3 be present at TFBS to set the stage for the transcription process? This study also revealed that the transcription-associated PTMs that occur in the tails of the histone $\mathrm{H} 3$ (H3K4me3 and H3K36me3) were indeed present in the protein-coding regions in the absence of HIRA (Goldberg et al. 2010). Since H3.3 was not present, then H3.1 and/or H3.2 containing appropriate marks are compensatory for H3.3, taking note that all three histone variants are virtually identical at their $\mathrm{N}$-terminal tails,
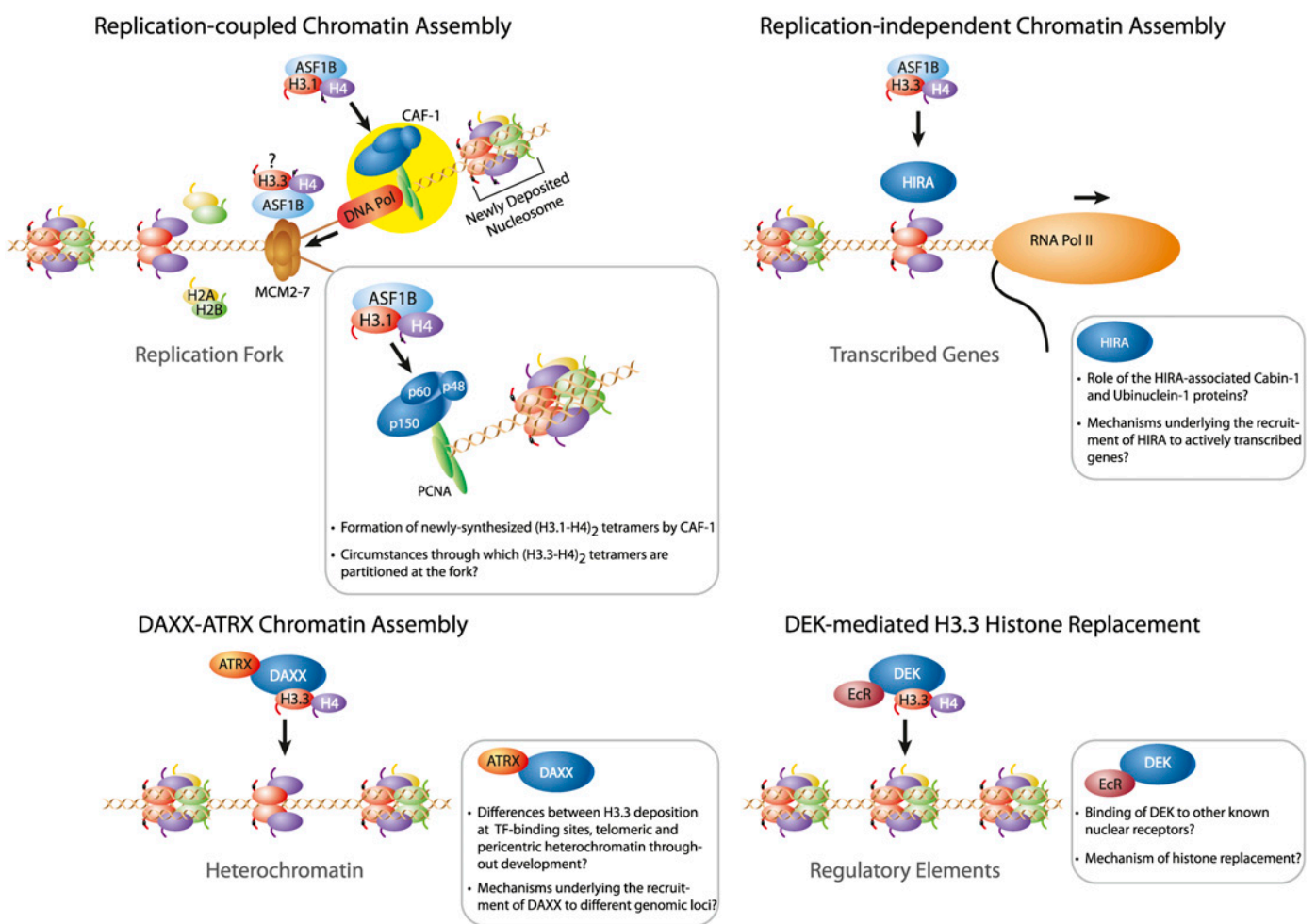

Figure 2. During RC assembly, the ASF1 chaperone is thought to transfer newly synthesized soluble H3.1-H4 dimers to CAF-1 through direct interactions with its p60 subunit (Tyler et al. 2001; Mello et al. 2002). CAF-1 would then facilitate the assembly of a central $(\mathrm{H} 3.1-\mathrm{H} 4)_{2}$ tetramer to which two H2A-H2B dimers are juxtaposed by other chaperones to complete a core nucleosomal unit. Similarly, during RI chromatin assembly, ASF1-bound H3.3-H4 would be transferred to HIRA for tetramer formation (Green et al. 2005). Note, however, that the deposition of H3.3 in the Drosophila male pronucleus is HIRA-dependent, but ASF1-independent (Bonnefoy et al. 2007) in the histone-rich fertilized egg. Novel alternate pathways for H3.3-H4 deposition include the DAXX chaperone coupled to the ATRX ATP-dependent chromatin remodeler (Drané et al. 2010; Goldberg et al. 2010), as well as targeted H3.3 deposition to regulatory elements by DEK (Sawatsubashi et al. 2010). Boxes highlight queries to consider in future studies. 
the targets for such modifications (Fig. 1). While overall transcription was operative, did the absence of $\mathrm{H} 3.3$ have any deleterious affects in this process-perhaps on elongation rates, for example? These findings challenge us to revisit the role(s) of histone $\mathrm{H} 3$ variants in transcription.

Using a different approach involving stringent biochemical analyses, Drané et al. (2010) found that DAXX and ATRX are tightly associated with histone H3.3, as opposed to histone $\mathrm{H} 3.1$, and are capable of depositing H3.3-H4 onto DNA in vitro, revealing the duo's chaperone status. Moreover, this study showed that DAXX is associated with H3.3 deposition in pericentromeric heterochromatin in mouse embryonic fibroblasts (MEFs). Yet, in $\mathrm{Daxx}^{-1-}$ cells, the DAXX-unattended H3.3 is found associated with NASP (nuclear autoantigenic sperm protein) and with the RC H3.1 chaperone CAF-1, reflecting some degree of flexibility to compensate for the lack of a given chaperone, yet this appears to default in the case of the whole organism given that DAXX defects lead to embryonic lethality in mice (Michaelson et al. 1999).

Whereas the vast majority of H3.3 is likely deposited by HIRA at protein-coding loci (Goldberg et al. 2010), DAXX is shown to direct H3.3 deposition at most genic and intergenic regulatory elements (Fig. 3; Goldberg et al. 2010). Moreover, ATRX targets DAXX to telomeres and ribosomal DNA for H3.3 deposition in ESCs (Wong et al. 2009; Goldberg et al. 2010), as well as pericentric heterochromatin in MEFs (Drané et al. 2010).

DAXX-ATRX-dependent H3.3 targeting to telomeres in ESCs appears to suppress transcription over telomeric repeats (Goldberg et al. 2010). ATRX is further found to promote $\mathrm{HP} 1 \alpha$ binding and maintenance of telomeric integrity, effects that dissipate as cells differentiate and establish more typical telomeric structures in which $\mathrm{H} 3.3$ levels are low (Wong et al. 2009). In contrast, DAXXATRX-dependent H3.3 targeting to pericentric chromatin is also reported to facilitate transcription of pericentric repeats in MEF cells (Drané et al. 2010). Given that DAXX has been shown to associate with HDACII and DEK (Hollenbach et al. 2002), and that ATRX recruits HP1 $\alpha$ (Lechner et al. 2005; Emelyanov et al. 2010), DAXX and ATRX clearly possess multiple roles in the regulation of chromatin assembly.

Along the same lines, the chromatin-bound protein DEK, common to all metazoans and some unicellular organisms (but not yeast), has been suggested to be a H3.3 histone chaperone in Drosophila and human cells (Sawatsubashi et al. 2010). Interestingly, this function seems to be impaired in the leukemia-related DEK-CAN fusion protein. The phosphorylated form of DEK is proposed to act as a transcriptional coactivator through direct interaction with the Drosophila nuclear ecdysone receptor (Sawatsubashi et al. 2010), potentially directing H3.3 deposition to regulatory elements to enhance transcription.

Why have cells evolved such chaperones that destine H3.3 localization to different genomic regions? Does this arise from differential targeting of the chaperones themselves? How interchangeable are these chaperones? Given that severe defects in HIRA or DAXX in mice are embryonic-lethal, and that the DEK-CAN fusion may be causally related in some AML patients, these chaperones cannot successfully rescue each other. Yet, in a defined phase exemplified by the given cell state studied, attempts to compensate are manifest: $\mathrm{H} 3$ post-translational marks associated with open chromatin and transcription are retained in coding regions devoid of $\mathrm{H} 3.3$ in $\mathrm{HirA}^{-/-}$ ESCs (Goldberg et al. 2010), H3.3 is deposited at TFBSs in the absence of ATRX (Goldberg et al. 2010), and salivary gland puffs still form without H3.3 deposition in Drosophila overexpressing a dominant-negative form of DEK (Sawatsubashi et al. 2010). Such plasticity is reminiscent of the case whereby the integrity of a repressive domain can be preserved. In the absence of SUV39H1/2, the levels of the repressive $\mathrm{H} 3 \mathrm{~K} 9 \mathrm{me} 3$ mark are depleted, yet the $\mathrm{H} 3 \mathrm{~K} 27 \mathrm{me} 1$ mark normally present with $\mathrm{H} 3 \mathrm{~K} 9 \mathrm{me} 3$ is

H3.3 Deposition on Chromosomes

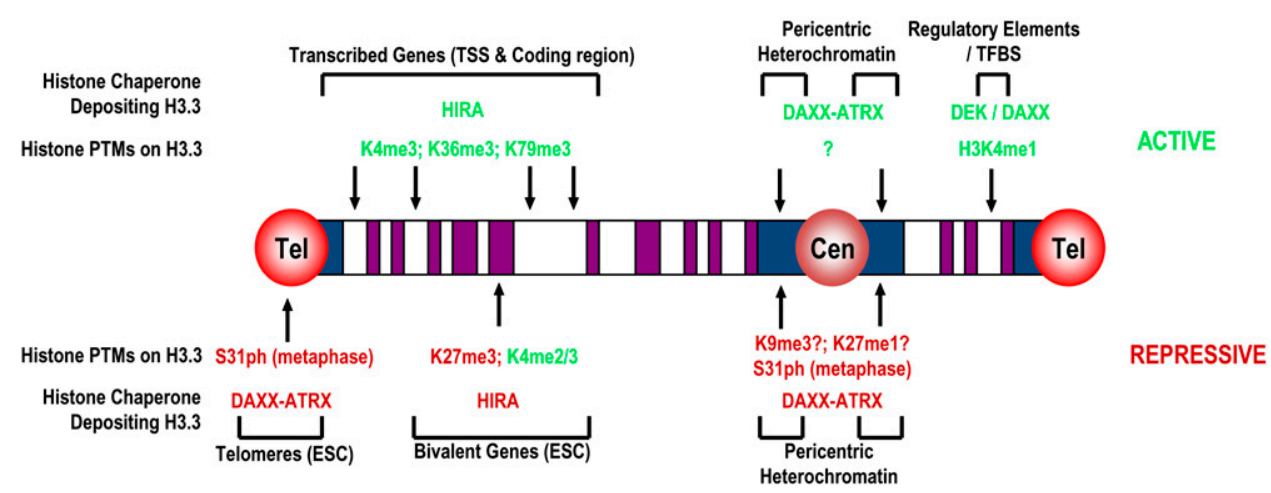

Figure 3. Model of H3.3 deposition by HIRA, DAXX, and DEK. HIRA deposits H3.3 within the coding region of transcribed genes, but also at the transcription start site (TSS) of both active and repressed high-CpG content genes in ESCs. Likewise, ATRX directs DAXXmediated H3.3 deposition to telomeres in ESCs, but also to pericentric heterochromatin. In Drosophila, phosphorylated DEK associates with the ecdysone receptor and acts as a coactivator. The H3.3 Ser 31 is phosphorylated in metaphase (Hake et al. 2005; Wong et al. 2009). 
converted to the repressive H3K27me3 mark, thereby compensating for this anomaly (Peters et al. 2003).

\section{Histone variants and inheritance}

Irrespective of the stage in the cell cycle at which the histone chaperones may act, the resultant chromatin domains will likely require establishment anew in future cell generations. Yet, the putative epigenetic inheritance of histone variants and/or their PTMs upon cell division remains a highly controversial and topical question. Based on somatic cell nuclear transplantation experiments in Xenopus, it has been proposed that the Lys 4 residue of the H3.3 variant may perpetuate epigenetic information for active transcription, independently of transcription $(\mathrm{Ng}$ and Gurdon 2008). Indeed, various models have been envisioned by which histone variants may help perpetuate epigenetic information.

An easily envisioned model of epigenetic inheritance involves splitting of parental $(\mathrm{H} 3-\mathrm{H} 4)_{2}$ tetramers. In favor of this model, it is well accepted that newly synthesized $(\mathrm{H} 3-\mathrm{H} 4)_{2}$ tetramers are assembled from $\mathrm{H} 3-\mathrm{H} 4$ dimeric precursors (Tagami et al. 2004). Furthermore, ASF1 copurifies with MCM proteins, key components of the replicative helicase, via histone contacts (Groth et al. 2007). Since ASF1 strictly binds H3-H4 dimers, and "parental" histone marks are detected in this complex when replication is halted by hydroxyurea (Jasencakova et al. 2010), ASF1 has been proposed to transiently partition nucleosomal $(\mathrm{H} 3-\mathrm{H} 4)_{2}$ tetramers facing the MCM2-7 replicative helicase. These findings further raised the question of whether histone PTMs may be transmitted in a "semiconservative" fashion as modified, pre-existing $\mathrm{H} 3-\mathrm{H} 4$ dimers formed tetramers with naïve, newly synthesized counterparts on replicated DNA. This supposition would require a concerted distribution of parental $\mathrm{H} 3-\mathrm{H} 4$ dimers to both leading and lagging strands.

However, there is also considerable evidence against this "semiconservative" theory. Past studies in various model systems have shown that global parental $(\mathrm{H} 3-\mathrm{H} 4)_{2}$ tetramers do not dissociate during replication (Annunziato 2005). Indeed, a recent and savvy approach distinguished newly formed $\mathrm{H} 3$ histones (expressed during $\mathrm{S}$ phase) from pre-existing ones through differential marking of each with an epitope tag and/or a heavy isotope via pulselabeling. The subsequent mass spectrometric analysis corroborated that, unlike H2A-H2B dimers, pre-existing $\mathrm{H} 3.1-\mathrm{H} 4$ histones by and large do not intermix with newly synthesized ones for at least two rounds of DNA replication (Xu et al. 2010). In contrast, approximately one-fifth of $\mathrm{H} 3.3-\mathrm{H} 4$ histones did intermix with newly synthesized ones after two rounds of replication, suggesting an appreciable level of tetramer disruption for nucleosomes encompassing this particular variant $\mathrm{Xu}$ et al. 2010). The significance of this observation with H3.3 remains to be elucidated, and may be limited to specific chromatin domains or histones. Yet it supports the finding that $\mathrm{H} 3.3$ histones form labile nucleosome structures when assembled with H2A.Z (Jin and Felsenfeld 2007), and likely facilitate transcription. In addition, these studies (Xu et al. 2010) strongly support the proposal that histone $\mathrm{H} 3.1$-associated modifications are likely transmitted to nearby nucleosomes, without tetramer disruption, through a mechanism analogous to the spreading of $\mathrm{H} 3 \mathrm{~K} 9 \mathrm{me} 3$ through HP1 binding and subsequent recruitment of the SUV39H1/2 enzyme that catalyzes this modification (Bannister et al. 2001; Lachner et al. 2001).

Since pre-existing histones distribute nearly equally to both leading and lagging strands (Annunziato 2005), an outstanding question is how a subset of histone PTMs are to be maintained within distinct chromatin domains. Indeed, various models by which histone PTMs are "duplicated" are now emerging. One advance in this regard involves the catalysis of $\mathrm{H} 3 \mathrm{~K} 27 \mathrm{me} 3$ mediated by the Ezh2 subunit of the PRC2 complex. In this case, Ezh2 activity is stimulated upon $\mathrm{H} 3 \mathrm{~K} 27 \mathrm{me} 3$ binding by another PRC2 component, EED (Margueron et al. 2009). Such allosteric activation would effectively offset the dilution of this "repressive" histone PTM post-replication, and maintain the chromatin environment. This type of propagation, similar to HP1-bound H3K9me3 and subsequent recruitment of SUV39H1/2 (Bannister et al. 2001; Lachner et al. 2001), may render the $(\mathrm{H} 3-\mathrm{H} 4)_{2}$ splitting question obsolete. Mechanistic studies examining the duplication of marks in vitro, such as $\mathrm{H} 3 \mathrm{~K} 27 \mathrm{me} 3$, are essential to resolving this issue. In the case of $\mathrm{H} 3 \mathrm{~K} 27 \mathrm{me} 3$, structural studies of the PRC2 complex may help to determine how internucleosomal contacts are made and thus propagate this PTM in the absence of hemimethylated $(\mathrm{H} 3-\mathrm{H} 4)_{2}$ tetramers, or determine whether it would be physically possible for the PRC2 complex to simultaneously bind and methylate single nucleosomes containing hemimethylated $(\mathrm{H} 3.3-\mathrm{H} 4)_{2}$ tetramers.

The new studies highlighted here have, without doubt, propelled the field forward. It is becoming increasingly clear that an array of histone chaperones specifically directs the deposition of histone variants at distinct chromatin loci throughout development and during the cell cycle. These studies also indicate that histone variants, like histone marks, do not strictly correlate with a clear transcriptional outcome, but rather contribute to the formation of specialized chromatin domains. The plasticity of the system highlights the intricacies involved, as well as the challenges ahead, to fully capture the workings of this process.

\section{Acknowledgments}

We thank Drs. Lynne Vales, Philip Voigt, and David Beck for discussions regarding this manuscript. E.I.C. is a recipient of a post-doctoral fellowship from the Natural Sciences and Engineering Research Council of Canada.

\section{References}

Annunziato AT. 2005. Split decision: What happens to nucleosomes during DNA replication? I Biol Chem 280: 1206512068.

Bannister AJ, Zegerman P, Partridge JF, Miska EA, Thomas JO, Allshire RC, Kouzarides T. 2001. Selective recognition of 
methylated lysine 9 on histone H3 by the HP1 chromo domain. Nature 410: 120-124.

Bonnefoy E, Orsi GA, Couble P, Loppin B. 2007. The essential role of Drosophila HIRA for de novo assembly of paternal chromatin at fertilization. PLoS Genet 3: 1991-2006.

Carroll D. 2008. Progress and prospects: Zinc-finger nucleases as gene therapy agents. Gene Ther 15: 1463-1468.

Drané P, Ouararhni K, Depaux A, Shuaib M, Hamiche A. 2010. The death-associated protein DAXX is a novel histone chaperone involved in the replication-independent deposition of H3.3. Genes Dev 24: 1253-1265.

Emelyanov AV, Konev AY, Vershilova E, and Fyodorov DV. 2010. Protein complex of Drosophila Atrx/Xnp and Hpla is required for the Formation of pericentric $\beta$-heterochromatin in vivo. I Biol Chem 285: 15027-15037

English CM, Adkins MW, Carson JJ, Churchill ME, Tyler JK. 2006. Structural basis for the histone chaperone activity of Asf1. Cell 127: 495-508.

Goldberg AD, Banaszynski LA, Noh KM, Lewis PW, Elsaesser SJ, Stadler S, Dewell S, Law M, Guo X, Li X, et al. 2010. Distinct factors control histone variant $\mathrm{H} 3.3$ localization at specific genomic regions. Cell 140: 678-691.

Green EM, Antczak AJ, Bailey AO, Franco AA, Wu KJ, Yates JR 3rd, Kaufman PD. 2005. Replication-independent histone deposition by the HIR complex and Asfl. Curr Biol 15: 2044 2049.

Groth A, Corpet A, Cook AJ, Roche D, Bartek J, Lukas J, Almouzni G. 2007. Regulation of replication fork progression through histone supply and demand. Science 318: 1928-1931.

Hake SB, Allis CD. 2006. Histone H3 variants and their potential role in indexing mammalian genomes: The ' $\mathrm{H} 3$ barcode hypothesis.' Proc Natl Acad Sci 103: 6428-6435.

Hake SB, Garcia BA, Kauer M, Baker SP, Shabanowitz J, Hunt DF, Allis CD. 2005. Serine 31 phosphorylation of histone variant $\mathrm{H} 3.3$ is specific to regions bordering centromeres in metaphase chromosomes. Proc Natl Acad Sci 102: 63446349.

Hollenbach AD, McPherson CJ, Mientjes EJ, Iyengar R, Grosveld G. 2002. Daxx and histone deacetylase II associate with chromatin through an interaction with core histones and the chromatin-associated protein Dek. J Cell Sci 115: 3319-3330.

Jasencakova Z, Scharf AN, Ask K, Corpet A, Imhof A, Almouzni G, Groth A. 2010. Replication stress interferes with histone recycling and predeposition marking of new histones. Mol Cell 37: 736-743.

Jin C, Felsenfeld G. 2007. Nucleosome stability mediated by histone variants H3.3 and H2A.Z. Genes Dev 21: 1519-1529.

Jin C, Zang C, Wei G, Cui K, Peng W, Zhao K, Felsenfeld G. 2009. H3.3/H2A.Z double variant-containing nucleosomes mark 'nucleosome-free regions' of active promoters and other regulatory regions. Nat Genet 41: 941-945.

Lachner M, O'Carroll D, Rea S, Mechtler K, Jenuwein T. 2001. Methylation of histone $\mathrm{H} 3$ lysine 9 creates a binding site for HP1 proteins. Nature 410: 116-120.

Lechner MS, Schultz DC, Negorev D, Maul GG, Rauscher FJ III. 2005. The mammalian heterochromatin protein 1 binds diverse nuclear proteins through a common motif that targets the chromoshadow domain. Biochem Biophys Res Commun 331: 929-937.

Loppin B, Bonnefoy E, Anselme C, Laurencon A, Karr TL, Couble P. 2005. The histone H3.3 chaperone HIRA is essential for chromatin assembly in the male pronucleus. Nature 437: 1386-1390.

Loyola A, Bonaldi T, Roche D, Imhof A, Almouzni G. 2006. PTMs on $\mathrm{H} 3$ variants before chromatin assembly potentiate their final epigenetic state. Mol Cell 24: 309-316.
Luger K, Mader AW, Richmond RK, Sargent DF, Richmond TJ. 1997. Crystal structure of the nucleosome core particle at 2.8 A resolution. Nature 389: 251-260.

Margueron R, Justin N, Ohno K, Sharpe ML, Son J, Drury WJ III, Voigt P, Martin SR, Taylor WR, De Marco V, et al. 2009. Role of the polycomb protein EED in the propagation of repressive histone marks. Nature 461: 762-767.

Mello JA, Sillje HH, Roche DM, Kirschner DB, Nigg EA, Almouzni G. 2002. Human Asf1 and CAF-1 interact and synergize in a repair-coupled nucleosome assembly pathway. EMBO Rep 3: 329-334.

Michaelson JS, Bader D, Kuo F, Kozak C, Leder P. 1999. Loss of Daxx, a promiscuously interacting protein, results in extensive apoptosis in early mouse development. Genes Dev 13: 1918-1923.

Moggs JG, Grandi P, Quivy JP, Jonsson ZO, Hubscher U, Becker PB, Almouzni G. 2000. A CAF-1-PCNA-mediated chromatin assembly pathway triggered by sensing DNA damage. Mol Cell Biol 20: 1206-1218.

Natsume R, Eitoku M, Akai Y, Sano N, Horikoshi M, Senda T. 2007. Structure and function of the histone chaperone CIA/ ASF1 complexed with histones H3 and H4. Nature 446: 338341.

$\mathrm{Ng}$ RK, Gurdon JB. 2008. Epigenetic memory of an active gene state depends on histone H3.3 incorporation into chromatin in the absence of transcription. Nat Cell Biol 10: 102-109.

Peters AH, Kubicek S, Mechtler K, O'Sullivan RJ, Derijck AA, Perez-Burgos L, Kohlmaier A, Opravil S, Tachibana M, Shinkai Y, et al. 2003. Partitioning and plasticity of repressive histone methylation states in mammalian chromatin. Mol Cell 12: 1577-1589.

Sawatsubashi S, Murata T, Lim J, Fujiki R, Ito S, Suzuki E, Tanabe M, Zhao Y, Kimura S, Fujiyama S, et al. 2010. A histone chaperone, DEK, transcriptionally coactivates a nuclear receptor. Genes Dev 24: 159-170.

Shibahara K, Stillman B. 1999. Replication-dependent marking of DNA by PCNA facilitates CAF-1-coupled inheritance of chromatin. Cell 96: 575-585.

Tagami H, Ray-Gallet D, Almouzni G, Nakatani Y. 2004. Histone H3.1 and H3.3 complexes mediate nucleosome assembly pathways dependent or independent of DNA synthesis. Cell 116: $51-61$.

Tyler JK, Collins KA, Prasad-Sinha J, Amiott E, Bulger M, Harte PJ, Kobayashi R, Kadonaga JT. 2001. Interaction between the Drosophila CAF-1 and ASF1 chromatin assembly factors. Mol Cell Biol 21: 6574-6584.

Wong LH, Ren H, Williams E, McGhie J, Ahn S, Sim M, Tam A, Earle E, Anderson MA, Mann J, et al. 2009. Histone H3.3 incorporation provides a unique and functionally essential telomeric chromatin in embryonic stem cells. Genome Res 19: 404-414.

Wong LH, McGhie JD, Sim M, Anderson MA, Ahn S, Hannan RD, George AJ, Morgan KA, Mann JR, Choo KH. 2010. ATRX interacts with $\mathrm{H} 3.3$ in maintaining telomere structural integrity in pluripotent embryonic stem cells. Genome Res 20: 351-360.

Xu M, Long C, Chen X, Huang C, Chen S, Zhu B. 2010. Partitioning of histone $\mathrm{H} 3-\mathrm{H} 4$ tetramers during DNA replication-dependent chromatin assembly. Science 328: 94-98.

Xue Y, Gibbons R, Yan Z, Yang D, McDowell TL, Sechi S, Qin J, Zhou S, Higgs D, Wang W. 2003. The ATRX syndrome protein forms a chromatin-remodeling complex with Daxx and localizes in promyelocytic leukemia nuclear bodies. Proc Natl Acad Sci 100: 10635-10640. 


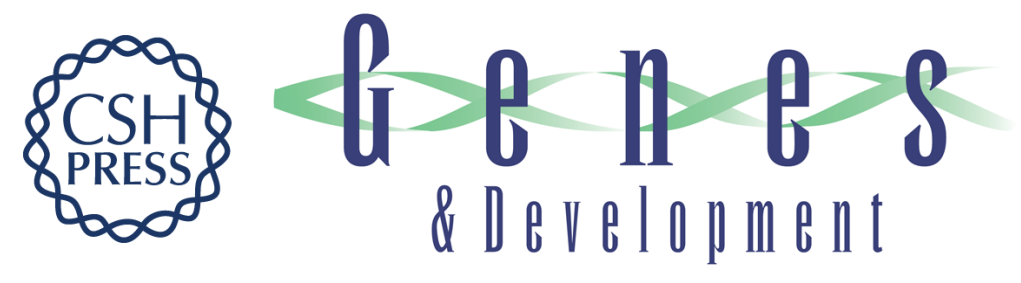

\section{New chaps in the histone chaperone arena}

Eric I. Campos and Danny Reinberg

Genes Dev. 2010, 24:

Access the most recent version at doi:10.1101/gad.1946810

Related Content The death-associated protein DAXX is a novel histone chaperone involved in the replication-independent deposition of $\mathrm{H} 3.3$

Pascal Drané, Khalid Ouararhni, Arnaud Depaux, et al.

Genes Dev. June, 2010 24: 1253-1265 A histone chaperone, DEK, transcriptionally coactivates a nuclear receptor

Shun Sawatsubashi, Takuya Murata, Jinseon Lim, et al.

Genes Dev. January, 2010 24: 159-170

References This article cites 36 articles, 17 of which can be accessed free at:

http://genesdev.cshlp.org/content/24/13/1334.full.html\#ref-list-1

Articles cited in:

http://genesdev.cshlp.org/content/24/13/1334.full.html\#related-urls

License Email Alerting
Service $\begin{aligned} & \text { Receive free email alerts when new articles cite this article - sign up in the box at the top } \\ & \text { right corner of the article or click here. }\end{aligned}$

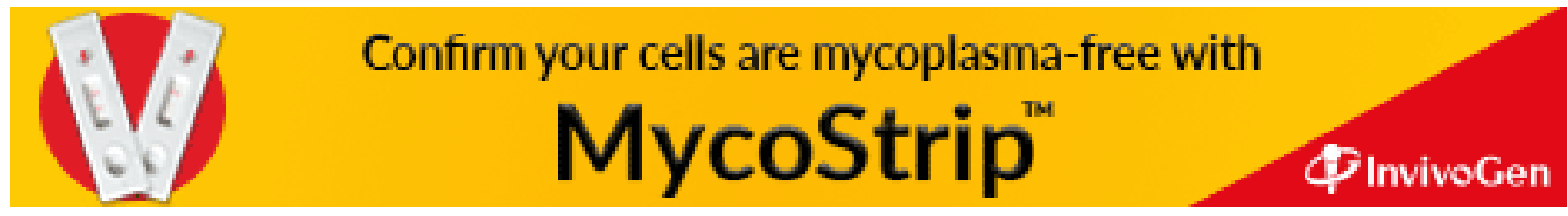

